\title{
Neurotoxicity of Paclitaxel and Rapamycin in a Rat Model with Transient Blood-Brain Barrier Opening
}

\author{
Won-Sang Cho, Jung Hoon Choi, ${ }^{2}$ O-Ki Kwon ${ }^{3}$ \\ Department of Neurosurgery, Seoul National University Hospital, Seoul, Korea \\ Department of Anatomy, ${ }^{2}$ College of Veterinary Medicine, Kangwon National University, Chuncheon, Korea \\ Department of Neurosurgery, ${ }^{3}$ Seoul National University Bundang Hospital, Seoul National University College of Medicine, Seongnam, Korea
}

Objective : Drug-eluting stents and balloons are occasionally used to reduce restenosis in medically intractable intracranial atherosclerotic stenosis. The authors aimed to determine whether such drugs can cause neurotoxicity due to local effects in a rat model.

Methods : Intra-arterial catheters were placed in the right common carotid artery of rats. Mannitol was injected to transiently open the brain-blood barrier (BBB), followed by high-dose drug (paclitaxel and rapamycin) injection. The optimal time interval of transient BBB opening for maximal drug penetration was determined to be 10 minutes. Paclitaxel and rapamycin were intraarterially administered in various doses. All the rats were neurologically evaluated, and their brain tissues were histologically examined.

Results : Neither neurological deficits nor histological abnormalities were observed in all the rats.

Conclusion : Paclitaxel and rapamycin did not cause neurotoxicity in a rat model with transient BBB opening.

Key Words : Intracranial atherosclerotic stenosis · Neurotoxicity · Paclitaxel · Rapamycin · Restenosis.

\section{INTRODUCTION}

Intracranial atherosclerotic stenosis (ICAS) is one of the causes of ischemic stroke. Recurrent stroke is known to occur in approximately $17 \%$ of patients with ICAS in 1 year, even with the best medical management ${ }^{2,4}$. Based on technical and clinical experience in the field of coronary artery diseases, endovascular treatment with stents has been attempted for patients with ICAS. Stenting in ICAS patients raises two major concerns : peri-procedural complications and high restenosis rates $^{2,4,8)}$. Regarding peri-procedural complications, recent studies have shown better outcomes than previous studies due to advancements in medical management, endovascular techniques and devices ${ }^{2,4,8)}$. Regarding the issue of high restenosis rates after cerebral arterial stenting, a proper solution has not yet been identified. Drug-eluting stents (DESs) and drug-eluting balloons (DEBs) in the coronary system, which release drugs that inhibit intimal hyperplasia, have also been used for ICAS with good preliminary results in reducing resteno$\operatorname{sis}^{1,2,6,7,9,10,12,13,16)}$. However, applying these drugs to cerebral ar-

- Received : April 1, $2021 ・$ Revised : May 28, $2021 ・$ Accepted : June 10, 2021

- Address for reprints : 0-Ki Kwon

Department of Neurosurgery, Seoul National University Bundang Hospital, 82 Gumi-ro 173beon-gil, Bundang-gu, Seongnam 13620, Korea

Tel : +82-31-787-7163, Fax : +82-31-787-4097, E-mail : meurokwonoki@gmail.com, ORCID : https://orcid.org/0000-0002-7379-9891

This is an Open Access article distributed under the terms of the Creative Commons Attribution Non-Commercial License (http://creativecommons.org/licenses/by-nc/4.0) which permits unrestricted non-commercial use, distribution, and reproduction in any medium, provided the original work is properly cited. 
teries may provoke neurotoxicity ${ }^{2,5,12)}$.

To our knowledge, no report has yet been published on this issue. The authors performed this study to evaluate neurotoxicity due to the local effects of high-dose paclitaxel and rapamycin using a rat model with transient blood-brain barrier (BBB) opening.

\section{MATERIALS AND METHODS}

The procedures for handling and caring for animals adhered to the guidelines that are in compliance with current international laws and policies (National Institute of Health Guide for the Care and Use of Laboratory Animals, Publication No. 85-23, 1985, revised 1996), and they were approved by the Institutional Animal Care and Use Committee of Kangwon National University (KIACUC-12-0014).

\section{Animal model, BBB opening and drug adminis- tration}

Male, specific pathogen-free, Sprague Dawley rats were obtained from Bio-Genomics (Seoul, Korea). The rats were 7-8 months old and weighed $250-350 \mathrm{~g}$. An intra-arterial infusion catheter was placed in the right common carotid artery.

To model the condition of a chronically ischemic brain with a partially disrupted BBB, temporary BBB opening was attempted via intra-arterial infusion of mannitol. A 20\% man-

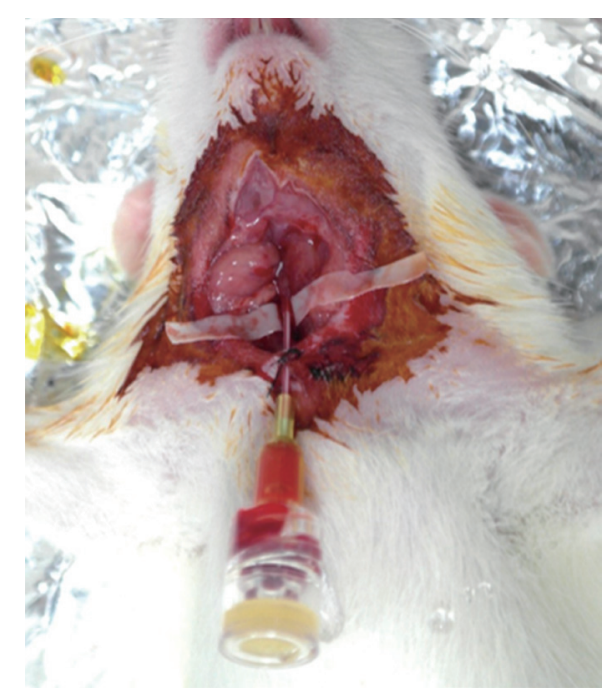

Fig. 1. A rat model for drug infusion via the right internal carotid artery with intra-arterial catheter indwelling. nitol solution (CJ Health Care, Daeso, Korea) was administered at a rate of $0.25 \mathrm{~mL} / \mathrm{kg} / \mathrm{sec}$ for 30 seconds with an infusion pump via the intra-arterial catheter. After mannitol infusion, a 2\% solution of Evans Blue (EB; Sigma, St. Louis, $\mathrm{MO}$, USA) in normal saline (4 $\mathrm{mL} / \mathrm{kg}$ of body weight) was injected via the intra-arterial catheter to confirm the degree of $\mathrm{BBB}$ opening in predetermined time intervals. Twenty-four hours after EB infusion, the whole rat brain was harvested, and the degree of EB extravasation was evaluated. A total of 25 rats (five rats per predetermined interval) were used for this purpose. Based on the degree of EB extravasation, the optimal time interval for transient BBB opening and maximal drug
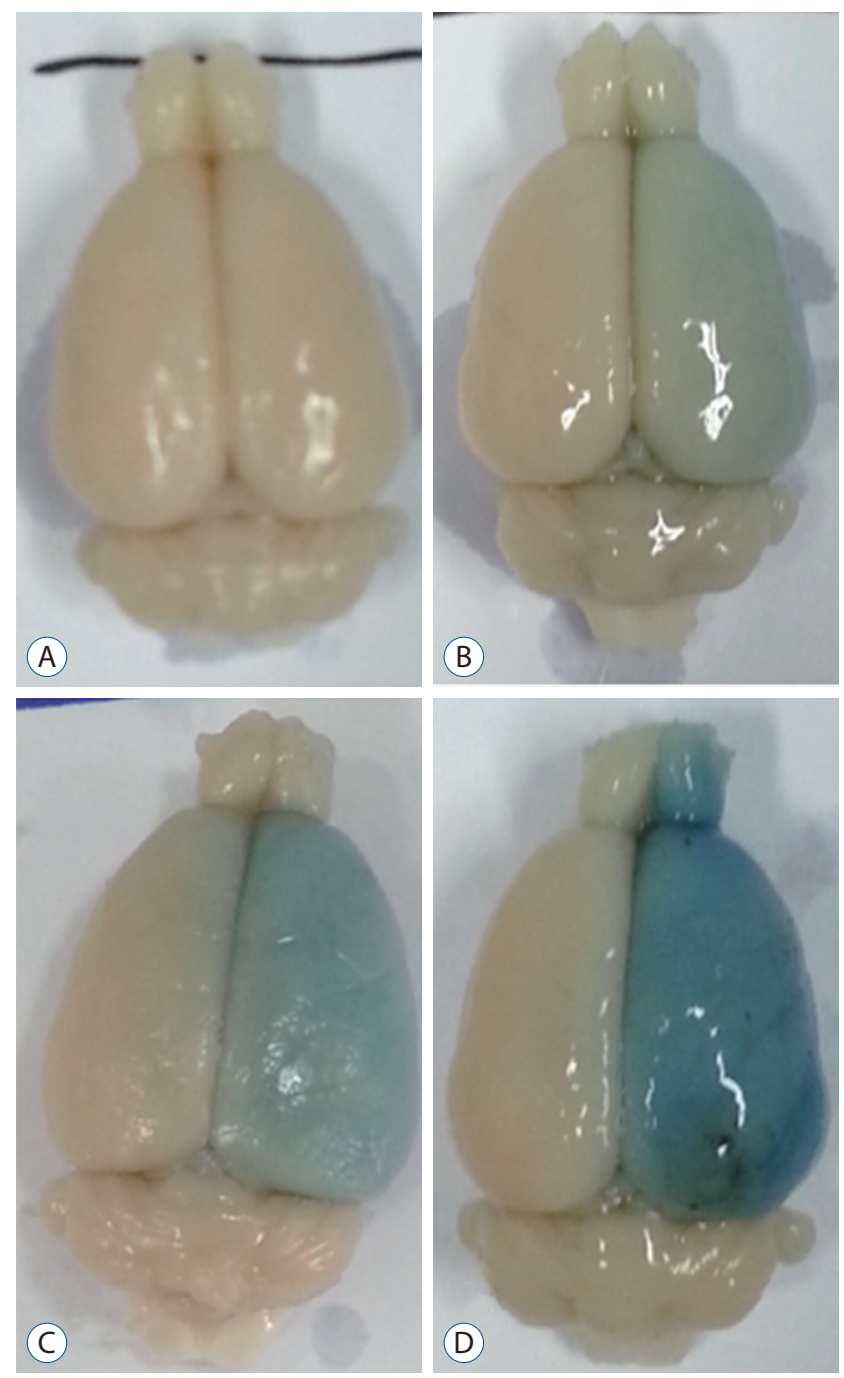

Fig. 2. Degree of extravasation of Evans Blue in the right hemispheres of rat brains. A : Grade 0 when there is no evidence of extravasation. B : Grade 1 when the degree of extravasation is pale. $C$ : Grade 2 when the degree is moderate. $\mathrm{D}$ : Grade 3 when the degree is strong. 
delivery was determined to be 10 minutes (Figs. 1-3).

The doses of paclitaxel and rapamycin (Sigma) were translated from humans into animals as equivalent doses using a formula based on body surface area : animal equivalent dose $(\mathrm{mg} / \mathrm{kg})=$ human dose $(\mathrm{mg} / \mathrm{kg}) \times$ human $\mathrm{K}_{\mathrm{m}} /$ animal $\left.\mathrm{K}_{\mathrm{m}}{ }^{14}\right)$. The drugs were injected as an intra-arterial bolus together with EB 10 minutes after mannitol administration. In the sham group, EB was infused without drugs. The brain tissues were obtained after 24 hours and 14 days. A total of 70 rats were used. Considering that the doses of the drugs coated on DESs and DEBs are 70-314 $\mu \mathrm{g}$ and 300-600 $\mu \mathrm{g}$, respectively ${ }^{18)}$, a human dose of $600 \mu \mathrm{g}$ was determined to be the maxi-

\begin{tabular}{lccccc}
\hline \multirow{2}{*}{ Rat No. } & \multicolumn{5}{c}{$\begin{array}{c}\text { Evans Blue injection time after mannitol administration } \\
\text { (minutes) }\end{array}$} \\
\cline { 2 - 6 } & $\mathbf{0}$ & $\mathbf{5}$ & $\mathbf{1 0}$ & $\mathbf{1 5}$ & $\mathbf{2 0}$ \\
\hline 1 & 0 & 0 & 1 & 1 & 0 \\
2 & 0 & 0 & 2 & 2 & 2 \\
3 & 2 & 0 & 1 & 1 & 3 \\
4 & 0 & 0 & 3 & 2 & 3 \\
5 & 0 & 3 & 2 & 1 & 3 \\
\hline
\end{tabular}

Fig. 3. Optimal time of blood-brain barrier opening after mannitol infusion. The degree of Evans Blue extravasation is colored from faint blue as grade 0 to dark blue as grade 3 . mum $^{2,4)}$. In this study, 1- to 4-times higher equivalent doses were tested.

\section{Neurological and histological examinations}

Neurological status was evaluated using Bederson et al. ${ }^{3)}$ 's grading. Frozen rat brains fixed with paraformaldehyde were serially sectioned on a cryostat into $30-\mu \mathrm{m}$ coronal sections. Histologic examination was performed with cresyl violet staining (Sigma) to assess neuronal death and Fluoro-Jade C (Histochem, Jefferson, AR, USA) histofluorescence staining to localize neuronal degeneration. Immunohistochemistry was performed with anti-glial fibrillary acidic protein (Biogenesis, San Ramon, CA, USA) for astrocytes and anti-ionized calcium-binding adapter molecule 1 (Wako, Osaka, Japan) for microglia. Detailed information is provided in the Supplement Method 1.

\section{RESULTS}

Most of rat brains were stained with EB, with a various range of grades from 1 to 3 (Supplementary Table 1). There were no histological abnormalities in the sham group (Supplementary Table 2, Supplementary Figs. 1 and 2). No neurotox-

\begin{tabular}{|c|c|c|c|c|c|c|c|c|c|c|c|c|c|}
\hline \multirow{4}{*}{ Day } & \multirow{4}{*}{$\begin{array}{l}\text { Rat } \\
\text { No. }\end{array}$} & \multicolumn{12}{|c|}{ Equivalent dose for human $(\mu \mathrm{g})$} \\
\hline & & \multicolumn{6}{|c|}{ Neuronal damage } & \multicolumn{6}{|c|}{ Inflammation } \\
\hline & & \multicolumn{3}{|c|}{ Striatum } & \multicolumn{3}{|c|}{ Hippocampus } & \multicolumn{3}{|c|}{ Striatum } & \multicolumn{3}{|c|}{ Hippocampus } \\
\hline & & 600 & 1200 & 2400 & 600 & 1200 & 2400 & 600 & 1200 & 2400 & 600 & 1200 & 2400 \\
\hline \multirow[t]{5}{*}{1} & 1 & 0 & 0 & 0 & 0 & 1 & 0 & 0 & 0 & 0 & 0 & 0 & 0 \\
\hline & 2 & 0 & 0 & 0 & 0 & 1 & 0 & 0 & 0 & 0 & 0 & 0 & 0 \\
\hline & 3 & 0 & 0 & 0 & 0 & 1 & 0 & 0 & 0 & 0 & 0 & 0 & 0 \\
\hline & 4 & 0 & 0 & 0 & 0 & 0 & 0 & 0 & 0 & 0 & 0 & 0 & 0 \\
\hline & 5 & 0 & 0 & 0 & 0 & 0 & 0 & 0 & 0 & 0 & 0 & 0 & 0 \\
\hline \multirow[t]{5}{*}{14} & 1 & 0 & 0 & 0 & 0 & 0 & 0 & 0 & 0 & 0 & 0 & 0 & 0 \\
\hline & 2 & 0 & 0 & 0 & 0 & 0 & 0 & 0 & 0 & 0 & 0 & 0 & 0 \\
\hline & 3 & 0 & 0 & 0 & 0 & 0 & 0 & 0 & 0 & 0 & 0 & 0 & 0 \\
\hline & 4 & 2 & 1 & 0 & 2 & 0 & 0 & 2 & 0 & 0 & 2 & 0 & 0 \\
\hline & 5 & 0 & 0 & 0 & 0 & 0 & 0 & 0 & 0 & 0 & 0 & 0 & 0 \\
\hline
\end{tabular}

(A)

Fig. 4. Histological change by paclitaxel (A) and rapamycin (B). The degree of neuronal damage and inflammation is colored from faint blue and orange hemisphere. 
icity was observed (Bederson's grade 0 in all cases) in any rats regardless of drug type, concentration or time period (Fig. 4 and Supplementary Figs. 3-14). Moderate degrees of inflammatory cell aggregation and neuronal damage were identified in 1 of 15 sections of just one rat brain (No. 4, day 14 after injection of $600 \mu \mathrm{g}$ human paclitaxel dose; Supplementary Figs. 15 and 16). No rat showed neurological abnormalities.

\section{DISCUSSION}

The restenosis rate after treating symptomatic ICAS with bare-metal stents is known to be as high as approximately $30 \%^{8,12)}$. Moreover, DESs and DEBs for ICAS caused lower rates of restenosis, ranging from $0 \%$ to $5 \%$ and from $0 \%$ to $13 \%$, respectively ${ }^{2,12}$. Using DESs and DEBs in cerebral arteries could provoke neurotoxicity. Fortunately, chronic exposure of the brain to low doses of the drugs released from DEBs and DESs appears safe, based on the lack of reports about neurotoxicity due to systemic effects of the drugs in interventional cardiology over 15 years. And, not a few preliminary preclinical and clinical studies showed no local and systemic complications, yet ${ }^{1,6,7,9,10,12,13,16)}$. In the early period after DES and DEB placement, high amounts of the drugs are released from the devices within the cerebral arteries. These high amounts could have harmful local effects on the brain. For example, $75-80 \%$ of the drugs are released into the bloodstream after DEB inflation, and only $10-15 \%$ are attached to the target vessel wall ${ }^{18}$. This study is the first animal experiment examining neurotoxicity due to the local effects of intra-arterial injection of high-dose paclitaxel and rapamycin. Our experiment showed no neurotoxicity, even though the drugs more easily penetrated into the brain tissues due to mannitol-induced $\mathrm{BBB}$ opening and higher doses, up to 4-times higher than the general dose on devices, were administered.

The major adverse event related to paclitaxel is peripheral neuropathy ${ }^{17)}$. It has been reported that peripheral neuropathy of any grade can occur with 100 to $300 \mathrm{mg} / \mathrm{m}^{2}$ paclitaxel. The cumulative dose that causes severe neuropathy is known to be approximately $1000 \mathrm{mg} / \mathrm{m}^{2}$. . For example, considering the surface dose $\left(3 \mu \mathrm{g} / \mathrm{mm}^{2}\right)$ and specifications of SeQuent ${ }^{\mathbb{R}}$ Please NEO (B. Braun Medical, Melsungen AG, Berlin, Germany), a novel paclitaxel-coated balloon ranging from 2.0 $\times 10$ $\mathrm{mm}$ to $4.0 \times 40 \mathrm{~mm}$, the total surface dose is approximately
$380 \mu \mathrm{g}-3 \mathrm{mg}$. As the total surface dose ranges between $1 / 180$ $1 / 60$ of the dose that causes peripheral neuropathy, paclitaxelrelated neurotoxicity hardly seems to occur, even if a full dose is released from the highest specification of the balloon. Moreover, paclitaxel cannot normally cross the BBB, and only two cases of grand mal seizure, leading to suspicion of central nervous system toxicity, have been reported ${ }^{15}$. Each ovarian cancer patient experienced seizures after intravenous injection of more than $250 \mathrm{mg} / \mathrm{m}^{2}$ paclitaxel in early clinical trials. However, the direct causality of paclitaxel is not clear because one patient had a brain metastasis and sub-therapeutic blood levels of phenytoin, and paclitaxel was not detected in the cerebrospinal fluid of the other patient.

Rapamycin is an immunosuppressant used for solid organ transplantation and a major drug coated on DESs. Rapamycin-related complications mainly include myelosuppression and hyperlipidemia. Although rapamycin easily crosses the $\mathrm{BBB}$, neurotoxicity has not been reported except in five cases of posterior reversible encephalopathy, which was suspected to be caused by rapamycin-related neurotoxicity simply based on recovery after rapamycin discontinuation ${ }^{5,11}$. Therefore, even though our experiment did not show high-dose rapamycininduced neurotoxicity in a rat model, using rapamycin-coated devices needs to be avoided until the adverse effects in humans are clearly shown.

There are a few limitations in this study. First, only two prototype of drugs for the DESs and DEBs were used. However, these data would be applicable because newer drugs are derivatives of rapamycin and paclitaxel. Second, it is an animal experiment. Third, the degree of BBB opening in rat models was a little inhomogeneous, however, there was no tendency of the degree of BBB opening according to some factors. The authors did not attempt additional experiments, sacrificing more animals. Further experiments in vivo or in vitro under the control of the degree of BBB opening can be necessary. Fourth, rats' neurological states were evaluated simply with a triage test which cannot detect minor changes.

\section{CONCLUSION}

There were no histological or neurological evidences of neurotoxicity by intra-arterial injection of paclitaxel and rapamycin in a rat model with transient BBB opening. This 
finding is expected to be helpful in consideration of the safety of drugs to reduce in-stent and post-procedural restenosis.

\section{CONFLICTS OF INTEREST}

No potential conflict of interest relevant to this article was reported.

\section{INFORMED CONSENT}

This type of study does not require informed consent.

\section{AUTHOR CONTRIBUTIONS}

\author{
Conceptualization : OKK \\ Data curation : WSC, JHC \\ Formal analysis: WSC, JHC \\ Funding acquisition : $\mathrm{OKK}$, WSC, JHC \\ Methodology : WSC, JHC \\ Project administration : WSC, OKK \\ Visualization : WSC, JHC \\ Writing - original draft : WSC, JHC, OKK \\ Writing - review \& editing : WSC, OKK
}

\section{ORCID}

$$
\begin{array}{ll}
\text { Won-Sang Cho } & \text { https://orcid.org/0000-0002-3345-8718 } \\
\text { Jung Hoon Choi } & \text { https://orcid.org/0000-0002-3725-4907 } \\
\text { O-Ki Kwon } & \text { https://orcid.org/0000-0002-7379-9891 }
\end{array}
$$

\section{- Acknowledgements}

This study was supported by a fund (02-2017-18) from Seoul National University Bundang Hospital.

\section{- Supplementary materials}

The online-only data supplement is available with this article at https://doi.org/10.3340/jkns.2021.0077.

\section{References}

1. Abou-Chebl A, Bashir Q, Yadav JS : Drug-eluting stents for the treatment of intracranial atherosclerosis: initial experience and midterm angiographic follow-up. Stroke 36 : e165-e168, 2005

2. Barnard ZR, Alexander MJ : Update in the treatment of intracranial atherosclerotic disease. Stroke Vasc Neurol 5 : 59-64, 2019

3. Bederson JB, Pitts LH, Tsuji M, Nishimura MC, Davis RL, Bartkowski H : Rat middle cerebral artery occlusion: evaluation of the model and development of a neurologic examination. Stroke 17 : 472-476, 1986

4. Chimowitz MI, Lynn MJ, Derdeyn CP, Turan TN, Fiorella D, Lane BF, et al. : Stenting versus aggressive medical therapy for intracranial arterial stenosis. N Engl J Med 365 : 993-1003, 2011

5. Gheith O, Cerna M, Halim MA, Nampoory N, Al-Otaibi T, Nair P, et al. : Sirolimus-induced combined posterior reversible encephalopathy syndrome and lymphocytic pneumonitis in a renal transplant recipient: case report and review of the literature. Exp Clin Transplant 15(Suppl 1) : 170-174, 2017

6. Gupta R, Al-Ali F, Thomas AJ, Horowitz MB, Barrow T, Vora NA, et al. : Safety, feasibility, and short-term follow-up of drug-eluting stent placement in the intracranial and extracranial circulation. Stroke 37 : 25622566, 2006

7. Kim J, Ban SP, Kim YD, Kwon OK : Long-term outcomes of drug-eluting stent implantation in patients with symptomatic extra- and intracranial atherosclerotic stenoses. J Cerebrovasc Endovasc Neurosurg 22 : 216-224, 2020

8. Kim SJ, Kim YJ, Ko JH : Long term outcome of in-stent stenosis after stent assisted coil embolization for cerebral aneurysm. J Korean Neurosurg Soc 62 : 536-544, 2019

9. Levy El, Hanel RA, Howington JU, Nemes B, Boulos AS, Tio FO, et al. : Sirolimus-eluting stents in the canine cerebral vasculature: a prospective, randomized, blinded assessment of safety and vessel response. J Neurosurg $100: 688-694,2004$

10. Levy El, Hanel RA, Tio FO, Garlick DS, Bailey L, Cunningham MR, et al. : Safety and pharmacokinetics of sirolimus-eluting stents in the canine cerebral vasculature: 180 day assessment. Neurosurgery 59 : 925-933; discussion 933-934, 2006

11. Maramattom BV, Wijdicks EF : Sirolimus may not cause neurotoxicity in kidney and liver transplant recipients. Neurology 63 : 1958-1959, 2004

12. Park S, Lee DG, Chung WJ, Lee DH, Suh DC : Long-term outcomes of drug-eluting stents in symptomatic intracranial stenosis. Neurointervention $8:$ 9-14, 2013

13. Qureshi Al, Kirmani JF, Hussein HM, Harris-Lane P, Divani AA, Suri MF, et al. : Early and intermediate-term outcomes with drug-eluting stents in high-risk patients with symptomatic intracranial stenosis. Neurosurgery 59 : 1044-1051; discussion 1051, 2006

14. Reagan-Shaw $S$, Nihal $M, A$ hmad $N$ : Dose translation from animal to human studies revisited. FASEB J 22 : 659-661, 2008

15. Rowinsky EK, Eisenhauer EA, Chaudhry V, Arbuck SG, Donehower RC : Clinical toxicities encountered with paclitaxel (taxol). Semin Oncol 
Neurotoxicity of Paclitaxel and Rapamycin | Cho WS, et al.

20(4 Suppl 3) : 1-15, 1993

16. Steinfort $B, N g P P$, Faulder $K$, Harrington $T$, Grinnell $V$, Sorby W, et al. : Midterm outcomes of paclitaxel-eluting stents for the treatment of intracranial posterior circulation stenoses. J Neurosurg 106 : 222-225, 2007
17. Velasco R, Bruna J : Taxane-induced peripheral neurotoxicity. Toxics 3 : 152-169, 2015

18. Waksman R, Pakala R : Drug-eluting balloon: the comeback kid? Circ Cardiovasc Interv 2 : 352-358, 2009 